\title{
Association of the TCF7L2 polymorphism with colorectal cancer and adenoma risk
}

\author{
Aditi Hazra ${ }^{1}$, Charles S. Fuchs ${ }^{2,3}$, Andrew T. Chan ${ }^{2}$, Edward L. Giovannucci ${ }^{2,4}$, and David \\ J. Hunter ${ }^{1,2,4}$ \\ ${ }^{1}$ Program in Molecular and Genetic Epidemiology, Department of Epidemiology, Harvard School of \\ Public Health, Boston, MA 02115 \\ ${ }^{2}$ Channing Laboratory, Department of Medicine, Brigham and Women's Hospital and Harvard \\ Medical School, 181 Longwood Avenue, Boston, MA 02115 \\ ${ }^{3}$ Department of Medical Oncology, Dana-Farber Cancer Institute, Boston, MA 02115 \\ ${ }^{4}$ Department of Nutrition, Harvard School of Public Health, Boston, MA 02115
}

\begin{abstract}
Interaction of $T C F 7 l 2$ with translocated $\beta$-catenin in the nucleus transiently converts $T C F 7 L 2$ to transcription factor activators, which induce the expression of target genes, including cyclin D1 and $c$-myc, in colorectal carcinogenesis. We evaluated the association of a polymorphism in TCF7L2 (RS12255372) which previously has been strongly associated with risk of Type II Diabetes, with colorectal cancer (CRC) and adenoma in the prospective Nurses' Health Study (NHS) and Health Professionals Follow-up Study (HPFS) cohorts. In the NHS and HPFS control populations, the TCF7L2 T-allele frequency ranged from 28 to $30 \%$ and the genotype distribution was in agreement with Hardy-Weinberg equilibrium. Overall, there was suggestive evidence for an inverse association associated with homozygosity for the minor allele of RS12255372 (TCF7L2 TT) and CRC (conditional and covariate adjusted $\mathrm{OR}=0.63,95 \% \mathrm{CI}$ : $0.37-1.08$; $\mathrm{P}$ for heterogeneity 0.52 for the association in women and men), which was more evident among women (OR=0.39, 95\%CI: 0.16 0.91). The polymorphism was not associated with risk of colorectal adenoma. Furthermore, we observed no evidence of effect modification between the TCF7L2 SNP and covariates such as family history (p-interaction $=0.45$ ) or BMI (p-interaction $=0.27$ ) or with genetic variants in the $A P C$ Asp1822 Val SNP (NHS cancer p-interaction=0.40, NHS adenoma p-interaction 0.10). In summary, the marginal association of TCF7L2 SNP with CRC may be due to chance, but warrants further laboratory and epidemiological investigation.
\end{abstract}

\section{Keywords}

TCF7L2; polymorphism; Wnt pathway; colorectal cancer

\section{Introduction}

T-cell factor (TCF)/LEF proteins facilitate the formation of multiprotein enhancer complexes to modulate the transcription of target genes. Interaction of T-cell factor 7 like 2 (TCF7L2, previously reported as $T C F-4$ ) with translocated $\beta$-catenin in the nucleus transiently converts $T C F 7 L 2$ to a transcription factor activator, which induces the expression of target genes,

To whom correspondence should be addressed: Aditi Hazra, Channing Laboratory, 181 Longwood Ave, Boston, MA 02115, Phone: 617-525-2035; Fax: 617-525-2008, Email: E-mail: ahazra@hsph.harvard.edu. 
including cyclin D1 and c-myc, in colorectal carcinogenesis [1]. Duval et al. (1999; 2000) characterized the genomic structure of TCF7L2/TCF-4 in colorectal cancer (CRC) cell lines [2-3]. TCF7L2 has alternative exons leading to isoforms with short, medium, or long Cterminal ends; the long $\mathrm{C}$-terminal ends may mediate transcriptional repression [3]. TCF7L2 frameshift mutation in MSI-H CRC leads to selective loss of TCF7L2 isoforms with CtBPbinding abilities [4]. Recently, a genome-wide analysis of 11 CRC tumor cell lines or xenografts classified TCF7L2 [5] as a cancer candidate gene (with a cancer mutation prevalance, CaMP, score of $2.8 ;>1$ is a cancer candidate gene). Polymorphisms in TCF7L2 have been associated with disease susceptibility. Specifically, Grant et al. (2006) demonstrated that a polymorphism in TCF7L2 is associated with increased risk of type II diabetes (T2D) [6-12] by impaired beta-cell function [7,9]. Epidemiologic studies have suggested that hyperinsulinemia may be related to the risk of colon adenoma and cancer [13-16]. Given its relevance to the etiology of colorectal cancer, we evaluated the association of the TCF7L2 (RS12255372) polymorphism with colorectal cancer and adenoma in the prospective Nurses' Health Study (NHS) and Health Professionals Follow-up Study (HPFS) cohorts.

\section{Materials and methods}

\section{Study population}

The NHS began in 1976 when 121,700 female registered nurses in the United States between the ages of 30 and 55 completed a self-administered questionnaire on their medical history and baseline health-related exposures. Subsequently, these participants have completed a mailed, self-administered questionnaire biennially to update information on their lifestyle, medical history, and diet (every 2 to 4 years).

In 1989 and 1990, 32,826 women, between the ages of 43 and 69, provided blood samples. Upon receipt, samples were immediately centrifuged and aliquoted into plasma, red blood cells, and buffy coat fractions for storage in liquid nitrogen. After blood collection through June 2000, 197 incident cases of colorectal cancer were confirmed through medical records or death reports, of which 190 cases were successfully genotyped. Controls were randomly selected from women who were alive and free of cancer at the time of case ascertainment. One control was matched to each case on year of birth and month of blood draw. As previously described, [17], cases of colorectal adenoma and controls were chosen from among women who supplied a blood sample, had a sigmoidoscopy or colonoscopy (by 1998) after providing a blood sample, and were free from diagnosed cancer (except nonmelanoma skin cancer), ulcerative colitis, and adenoma before endoscopy. Newly diagnosed polyps (in individuals who never had a polyp diagnosed before the date of blood draw) were reported on the 1990, 1992, 1994, 1996, or 1998 questionnaire. These polyps were confirmed to be adenomatous by review of histopathological reports and were classified by location (proximal or distal colon, or rectum), size ( $<1 \mathrm{~cm}, \geq 1$ $\mathrm{cm}$ ), and histology (tubular, tubulovillous, villous, carcinoma-in-situ (CIS)) by study investigators blinded to the exposure information. Advanced adenoma was defined as large adenomas $\geq 1 \mathrm{~cm}$ in size and [Aditi - do you mean "or" as well here - typically advanced adenoma also includes polyps $<1 \mathrm{~cm}$ with any villous component) tubulovillous, villous, or carcinoma in situ histology. Between 1989 and 1998, 557 women with colorectal adenoma were identified, and one control was selected for each case, matched on year of birth, year of blood draw, time period of recent endoscopy, indication for endoscopy, and time period of first or most recent endoscopy. One case was subsequently found to be hyperplastic and was excluded from the analysis. Thus, the total number of cases and controls analyzed in this study were 556 cases and 557 controls. Diet was assessed in 1986, 1990, 1994 and 1996 with a semiquantitative food frequency questionnaire.

The HPFS began in 1986 when 51,529 US male dentists, optometrists, osteopaths, podiatrists, pharmacists, and veterinarians, ages 40 to 75 years, responded to a mailed questionnaire [18]. 
These men provided baseline information on age, marital status, height, weight, ancestry, medications, smoking history, medical history, physical activity, and diet. Exposure and medical history information are updated every 2 years. Blood samples were collected from 18,225 of the HPFS participants between 1993 and 1995. Among these men, 168 incident cases of CRC were identified between the date of blood draw and January 2002. Men who were alive and free of diagnosed cancer at the time of case ascertainment were selected as controls and were matched to cases on year of birth and month of blood draw.

\section{Genotyping methods}

Genotyping was performed at the Dana-Farber/Harvard Cancer Center High-Throughput Polymorphism Core. DNA was extracted from $50-\mu \mathrm{L}$ buffy coat fractions diluted with $150 \mu \mathrm{L}$ of PBS by the Qiagen QIAamp Blood Kit (Qiagen, Chatsworth, CA) spin protocol. The genotype of theTCF7L2 polymorphism was determined by measuring end-point fluorescence using the 5' nuclease assay (Taqman) on the ABI PRISM 7900HT Sequence Detection System (Applied Biosystems, Foster City, CA) [19]. The primers for RS12255372 (G,T) in Intron 3 TCF7L2 were: Forward: GCTGAGCTGCCCAGGAATAT; Reverse: GCAGAGGCCTGAGTAATTATCAGAA.

Quality control was ensured by including a random $10 \%$ of the samples in the 96-well plates as duplicates. The quality control samples served as internal controls to validate the genotyping methods; there was $100 \%$ concordance of the quality control samples. Laboratory personnel were blinded to the status (case, control, or quality control) of samples.

\section{Statistical analysis}

The study population included 357 incident CRC cases and 814 matched controls and 556 incident adenoma cases and 557 matched controls for the analyses. The number of cases and controls for the polymorphism was slightly lower because of missing genotyping data. Genotype distributions were evaluated for agreement with Hardy-Weinberg equilibrium (HWE) by the chi-square test. We evaluated differences among cases and controls using the chi-square test for categorical variables and the paired t-test for continuous variables.

We used both conditional and unconditional logistic regression for the analyses to compute genotype odds ratios (ORs), 95\% confidence intervals (CIs), allelic trend tests, and interactions. In the multivariate models, we adjusted for known risk factors for colorectal neoplasia, including family history of CRC, pack-years of smoking, postmenopausal hormone use, body mass index (BMI), physical activity, aspirin use, total energy intake, and consumption of red meat, alcohol, and folate. Unconditional logistic analyses explicitly adjusted for matching variables, including age, history of previous endoscopy (before blood draw), year of endoscopy, (endoscopy variables I assume are only for the polyps) and blood draw factors.

The SNP was initially examined with the codominant model, evaluating the TCF7L2 as a threelevel categorical variable. We also used the dominant model, comparing variant carriers with the referent homozygous wild type. The risk for advanced and small adenoma was evaluated by polytomous logistic regression with an ordinal outcome variable that was modeled as a three-level categorical variable: advanced adenoma, small adenoma, and controls. Effect modification by covariates, family history, and BMI was tested with p-values obtained from the likelihood ratio test comparing models with and without the interaction term between genotype and covariate. Gene-gene interactions with a non-synonymous Adematous Polyposis Coli (APC) Asp $1822 \mathrm{Val}$ were tested by likelihood ratio tests comparing the model with main effects for each polymorphism to the model including indicator variables for the crosstabulation of the two polymorphisms. All p-values reported are two-sided. 


\section{Results \\ Cancer}

Details of this study population have been previously reported [17]. Briefly, there were 367 incident cases of CRC and 852 controls. The mean age of cases and their matched controls was 68 years. As expected, family history was more common in the cases, whereas aspirin use, multivitamin use, and being a never smoker was more common among controls.

\section{Adenoma}

Details of this study population have been previously reported [18]. The mean age of cases and their matched controls was 62.3 years. Family history of CRC, smoking $>25$ pack-years, BMI $>30 \mathrm{~kg} / \mathrm{m}^{2}$, and consumption of $>1$ serving of red meat/day were associated with an increased risk of colorectal adenoma. In addition, cases were less likely than controls to be current users of postmenopausal hormones or to take multivitamins and aspirin ( $<7$ aspirin tablets/week). A sub-analysis of distal-only adenoma did not reveal a point estimate that varied (point estimate varied less than 10\%) from the overall main effect of the SNP.

\section{TCF7L2 polymorphism}

The TCF7L2 T-allele frequency ranged from 28 to $30 \%$ and the genotype distribution was in agreement with Hardy-Weinberg equilibrium. Overall, there was a marginal inverse association with the minor allele of RS12255372 (TCF7L2 TT) and CRC (conditional and covariate adjusted $\mathrm{OR}=0.63,95 \% \mathrm{CI}$ : $0.37-1.08$; $\mathrm{P}$ for heterogeneity 0.52 for the association in women and men). Although limited by sample size and thus perhaps due to chance, this association was more evident among women $(\mathrm{OR}=0.39,95 \% \mathrm{CI}$ : $0.16-0.91)$. The TCF7L2 polymorphism was not associated with risk of small (multivariate OR=0.95 (95\% CI: 0.74 $1.18)$ ) or advanced (multivariate $\mathrm{OR}=1.01$ (95\% CI: $0.78-1.32)$ ) colorectal adenomas (pvalue $=0.82$ for 2 - degree of freedom likelihood ratio test, data not shown). Furthermore, we observed no evidence of effect modification between the TCF7L2 SNP and covariates such as family history (p-interaction $=0.45$ ) and BMI (p-interaction $=0.27$ ) or with genetic variants in the APC Asp1822 Val SNP (NHS cancer p-interaction=0.40, NHS adenoma p-interaction 0.10).

\section{Discussion}

$A P C$ is the gatekeeper tumor suppressor gene associated with CRC. High-penetrance mutations in the APC pathway are a hallmark of familial CRC (familial adenomatus polyposis or FAP). In this pathway, activated $W n t$ signaling leads to the accumulation of nuclear $\beta$-catenin. The cytoplasmic level of $\beta$-catenin is regulated by ubiquitin-mediated proteolysis after it is targeted

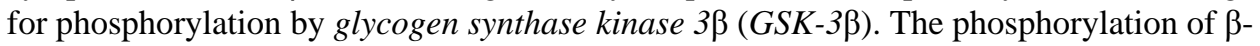
catenin by $G S K-3 \beta$ occurs in the context of a structural complex that includes Axin and APC [20]. GSK-3 $\beta$ activity is inhibited in the presence of a Wnt signal [21]. Interaction of excess translocated $\beta$-catenin in the nucleus with TCF7L2 upregulates expression of specific target genes, including cyclin D1, c-myc [22-23] and GLP-1 [24].

In this study, we observed a marginal inverse association between the TCF7L2 polymorphism and risk of CRC, but not adenoma. Evaluating the gene-environment association of the SNP with family history and BMI did not reveal a significant interaction. Furthermore, the APC Asp1822 Val SNP did not modify the risk of CRC and adenoma among women for any TCF7L2 genotype.

In the past year several publications have reported an increased risk of T2D (this abbrevation, if you are going to use it, needs to be defined earlier) among $T$-allele carriers. Grant et al. (2006) identified DG10S478 located in intron 3-exon 4 of the TCF7L2 gene on chromosome 10q25.2 
(in a well-defined LD block of $92.1 \mathrm{~kb}$ based on CEU HapMap v16; GenBank NM 030756) that had an $\mathrm{r}^{2}$ of 0.95 with the rs12255372 SNP [6]. The authors suggest that the TCF7L2 variants increase T2D risk by altering proglucagon gene expression [6] in enteroendrocrine cells [24] via the Wnt signaling pathway [26]. Florez et al. (2006) reported that individuals with the $T T$ genotype have decreased insulin secretion [7]. The reduced insulin secretion may be due to decreased expression of proglucagon and glucagons like peptide-1 [24]. Zhang et al. (2006) reported an increased risk for T2D for the TT genotype in the NHS (687 T2D, 1051 controls) with an OR of 1.86 (95\% CI: $1.30-2.67$ ) and in the HPFS (886 T2D, 896 controls) with an OR of 2.15 (95\% CI: 1.48 - 3.13) [8]. A study of breast cancer risk among 592 German families [25] and 735 controls reported a modest association with increased risk (OR=1.19 (95\% CI: 1.01-1.42) for the $T$-allele.

I might include a paragraph here which discussed the assocation between diabetes and CRC and how you would have expected, based on that relationship, to have seen an increased risk with the polymorphism. Hence, this study suggests that there is NO increased risk and possibly an inverse association that warrants more investigation. That might be a better sell overall as a null study which if anything, shows inverse assocation.

The TCF7L2 polymorphism is located in interspersed repeat elements, $41 \mathrm{~kb}$ upstream and 9 $\mathrm{kb}$ downstream of alternatively spliced exon 4 [7]. A limitation of this analysis is that the functional relevance of this SNP is unknown. In summary, the marginal inverse association of TCF7L2 SNP with CRC may be due to chance, but warrants further laboratory and epidemiological investigation.

\section{Glossary}

The commonly used abbreviations are

SNPs single nucleotide polymorphisms

\section{TCF7L2}

$$
\text { T-cell factor } 7 \text { like } 2
$$

CRC

$$
\text { colorectal cancer }
$$

T2D

Type II Diabetes

NHS

Nurses' Health Study

HPFS

Health Professionals Follow-up Study

OR

Odds Ratio

CI

confidence interval

\section{References}

1. Nelson WJ, Nusse R. Convergence of Wnt, beta-catenin, and cadherin pathways. Science 2004;303:1483-1487. [PubMed: 15001769] 
2. Duval A, Gayet J, Zhou XP, Iacopetta B, Thomas G, Hamelin R. Frequent frameshift mutations of the TCF-4 gene in colorectal cancers with microsatellite instability. Cancer Res 1999;59:4213-4215. [PubMed: 10485457]

3. Duval A, Rolland S, Tubacher E, Bui H, Thomas G, Hamelin R. The human T-cell transcription factor-4 gene: structure, extensive characterization of alternative splicings, and mutational analysis in colorectal cancer cell lines. Cancer Res 2000;60:3872-3879. [PubMed: 10919662]

4. Cuilliere-Dartigues P, El-Bchiri J, Krimi A, Buhard O, Fontanges P, Flejou JF, Hamelin R, Duval A. TCF-4 isoforms absent in TCF-4 mutated MSI-H colorectal cancer cells colocalize with nuclear CtBP and repress TCF-4-mediated transcription. Oncogene 2006;25:4441-4448. [PubMed: 16547505]

5. Sjölblom, et al. The consensus coding sequences of human breast and colorectal cancers. Science 2006;314:268-274. [PubMed: 16959974]

6. Grant SF, Thorleifsson G, Reynisdottir I, Benediktsson R, Manolescu A, Sainz J, Helgason A, Stefansson H, Emilsson V, Helgadottir A, Styrkarsdottir U, Magnusson KP, Walters GB, Palsdottir E, Jonsdottir T, Gudmundsdottir T, Gylfason A, Saemundsdottir J, Wilensky RL, Reilly MP, Rader DJ, Bagger Y, Christiansen C, Gudnason V, Sigurdsson G, Thorsteinsdottir U, Gulcher JR, Kong A, Stefansson K. Variant of transcription factor 7-like 2 (TCF7L2) gene confers risk of type 2 diabetes. Nat Genet 2006;38:320-323. [PubMed: 16415884]

7. Florez Florez JC, Jablonski KA, Bayley N, Pollin TI, de Bakker PIW, Shuldiner AR, Knowler WC, Nathan DM, Altshuler D. the Diabetes Prevention Program: TCF7L2 polymorphisms and progression to diabetes in the Diabetes Prevention Program. N Engl J Med 2006;355:241-250. [PubMed: 16855264]

8. Zhang C, Qi L, Hunter DJ, Meigs JB, Manson JE, van Dam RM, Hu FB. Variant of transcription factor 7-like 2 (TCF7L2) gene and the risk of type 2 diabetes in large cohorts of U.S. women and men. Diabetes 2006;55:2645-2648. [PubMed: 16936216]

9. Saxena R, Gianniny L, Burtt NP, Lyssenko V, Giuducci C, Sjogren M, Florez JC, Almgren P, Isomaa B, Orho-Melander M, Lindblad U, Daly MJ, Tuomi T, Hirschhorn JN, Ardlie KG, Groop LC, Altshuler D. Common single nucleotide polymorphisms in TCF7L2 are reproducibly associated with type 2 diabetes and reduce the insulin response to glucose in nondiabetic individuals. Diabetes 2006;55:28902895. [PubMed: 17003358]

10. Scott LJ, Bonnycastle LL, Willer CJ, Sprau AG, Jackson AU, Narisu N, Duren WL, Chines PS, Stringham HM, Erdos MR, Valle TT, Tuomilehto J, Bergman RN, Mohlke KL, Collins FS, Boehnke M. Association of transcription factor 7-like 2 (TCF7L2) variants with type 2 diabetes in a Finnish sample. Diabetes 2006;55:2649-2653. [PubMed: 16936217]

11. Groves CJ, Zeggini E, Minton J, Frayling TM, Weedon MN, Rayner NW, Hitman GA, Walker M, Wiltshire S, Hattersley AT, McCarthy MI. Association analysis of 6,736 U.K. subjects provides replication and confirms TCF7L2 as a type 2 diabetes susceptibility gene with a substantial effect on individual risk. Diabetes 2006;55:2640-2644. [PubMed: 16936215]

12. Damcott CM, Pollin TI, Reinhart LJ, Ott SH, Shen H, Silver KD, Mitchell BD, Shuldiner AR. Polymorphisms in the transcription factor 7-like 2 (TCF7L2) gene are associated with type 2 diabetes in the Amish: replication and evidence for a role in both insulin secretion and insulin resistance. Diabetes 2006;55:2654-2659. [PubMed: 16936218]

13. Platz EA, Hankinson SE, Rifai N, Colditz GA, Speizer FE, Giovannucci E. Glycosylated hemoglobin and risk of colorectal cancer and adenoma (United States). Cancer Causes Control 1999;10:379-386. [PubMed: 10530607]

14. Wei EK, Ma J, Pollak MN, et al. C-peptide, insulin-like growth factor binding protein-1, glycosylated hemoglobin, and the risk of distal colorectal adenoma in women. Cancer Epidemiol Biomarkers Prev 2006;15:750-755. [PubMed: 16614119]

15. Wei EK, Ma J, Pollak MN, et al. A prospective study of C-peptide, insulin-like growth factor-I, insulin-like growth factor binding protein-1, and the risk of colorectal cancer in women. Cancer Epidemiol Biomarkers Prev 2005;14:850-855. [PubMed: 15824155]

16. Tran TT, Naigamwalla D, Oprescu AI, et al. Hyperinsulinemia, but not other factors associated with insulin resistance, acutely enhances colorectal epithelial proliferation in vivo. Endocrinology 2006;147:1830-1837. [PubMed: 16410309] 
17. Koushik A, Kraft P, Fuchs C, Hankinson SE, Willett WC, Giovannucci EL, Hunter DJ. Polymorphisms in one-carbon metabolism genes and associations with colorectal cancer. Cancer Epidemiol. Biomarkers Prev 2006;15:2408-2417. [PubMed: 17164363]

18. Tranah GJ, Giovannucci E, Ma J, Fuchs C, Hunter DJ. APC Asp1822Val and Gly2502Ser polymorphisms and risk of colorectal cancer and adenoma. Cancer Epidemiol. Biomarkers Prev 2005; 14:863-870. [PubMed: 15824157]

19. Livak KJ. Allelic discrimination using fluorogenic probes and 5' nuclease assay. Genet Anal 1999;14:143-149. [PubMed: 10084106]

20. Hart MJ, de los Santos R, Albert IN, Rubinfeld B, Polakis P. Downregulation of beta-catenin by human Axin and its association with the APC tumor suppressor, beta-catenin and GSK3 beta. Curr Biol 1998;8:573-581. [PubMed: 9601641]

21. Polakis P. Wnt signaling and cancer. Genes Dev 2000;14:1837-1851. [PubMed: 10921899]

22. Tetsu O, McCormick F. Beta-catenin regulates expression of cyclin D1 in colon carcinoma cells. Nature 1999;398:422-426. [PubMed: 10201372]

23. He TC, Sparks AB, Rago C, Hermeking H, Zawel L, da Costa LT, Morin PJ, Vogelstein B, Kinzler KW. Identification of c-MYC as a target of the APC pathway. Science 1998 Sep 4;281(5382):15091512. [PubMed: 9727977]

24. Yi F, Brubaker PL, Jin T. TCF-4 mediates cell type-specific regulation of proglucagon gene expression by beta-catenin and glycogen synthase kinase-3beta. J Biol Chem 2005;280:1457-1464. [PubMed: 15525634]

25. Burwinkel B, Shanugam KS, Hemminki K, Meindl A, Schmutzler RK, Sutter C, Wappenschmidt B, Kiechle M, Bartram CR, Frank B. Transcription factor 7-like 2 (TCF7L2) variant is associated with familial breast cancer risk: a case-control study. BMC Cancer 2006;6:268. [PubMed: 17109766]

26. Smith U. TCF7L2 and type 2 diabetes - we WNT to know. Diabetologia 2007;50:5-7. [PubMed: 17102948] 
Table 1

Association between the TCF7L2 (rs12255372) genetic variants with colorectal cancer and adenoma risk in the Nurses' Health Study and Health Professionals Follow-up Study

\begin{tabular}{|c|c|c|c|c|}
\hline & $\begin{array}{l}\text { Cases } \\
\mathbf{N}(\%)\end{array}$ & $\begin{array}{l}\text { Controls* } \\
\text { N }(\%)\end{array}$ & $\begin{array}{l}\text { Conditional OR } \\
(95 \% \text { CI })\end{array}$ & $\begin{array}{l}\text { Multivariate } \\
\text { Conditional OR } \\
\text { (95\% CI) }\end{array}$ \\
\hline & \multicolumn{4}{|c|}{ NHS and HPFS CRC ${ }^{3}$} \\
\hline$T C F 7 L 2$ & $\mathrm{~N}=357$ & $\mathrm{~N}=814$ & & \\
\hline$G G$ & $199(55.7)$ & $413(50.7)$ & 1.00 (Reference) & 1.00 (Reference) \\
\hline$G T$ & $135(37.8)$ & $328(40.3)$ & $0.89(0.68-1.16)$ & $0.90(0.68-1.18)$ \\
\hline \multirow[t]{2}{*}{$T T$} & $23(6.4)$ & $73(9.0)$ & $0.64(0.38-1.07)$ & $0.63(0.37-1.08)$ \\
\hline & & & ${ }^{4} P$-trend & 0.10 \\
\hline \multirow[t]{2}{*}{$G T+T T$} & $158(44.3)$ & $401(49.3)$ & $0.84(0.69-1.03)$ & $0.84(0.68-1.04)$ \\
\hline & NHS CRC & & & \\
\hline \multicolumn{5}{|l|}{ TCF7L2 } \\
\hline$G G$ & $100(53.2)$ & $233(49.7)$ & 1.00 (Reference) & 1.00 (Reference) \\
\hline$G T$ & $81(43.1)$ & $194(41.4)$ & $0.99(0.70-1.40)$ & $1.00(0.68-1.46)$ \\
\hline \multirow[t]{2}{*}{$T T$} & $7(3.7)$ & $42(9.0)$ & $0.39(0.17-0.91)$ & $0.39(0.16-0.91)$ \\
\hline & & & $P$-trend & 0.14 \\
\hline \multirow[t]{2}{*}{$G T+T T$} & $88(46.8)$ & $236(50.4)$ & $0.81(0.62-1.07)$ & $0.81(0.60-1.07)$ \\
\hline & HPFS CRC & & & \\
\hline \multicolumn{5}{|l|}{ TCF L2 } \\
\hline$G G$ & $101(58.7)$ & $186(52.8)$ & 1.00 (Reference) & 1.00 (Reference) \\
\hline$G T$ & $55(32.0)$ & $135(38.4)$ & $0.75(0.50-1.13)$ & $0.78(0.50-1.20)$ \\
\hline \multirow[t]{2}{*}{$T T$} & $16(9.3)$ & $31(8.8)$ & $0.96(0.48-1.91)$ & $0.89(0.42-1.89)$ \\
\hline & & & $P$-trend & 0.37 \\
\hline \multirow[t]{2}{*}{$G T+T T$} & $71(41.3)$ & $166(47.2)$ & $0.88(0.66-1.17)$ & $0.87(0.64-1.18)$ \\
\hline & NHS Aden & & & \\
\hline \multicolumn{5}{|l|}{$T C F 7 L 2$} \\
\hline$G G$ & $271(51.8)$ & $273(52.4)$ & 1.00 (Reference) & 1.00 (Reference) \\
\hline$G T$ & $218(41.7)$ & $204(39.2)$ & $1.06(0.81-1.39)$ & $1.12(0.83-1.51)$ \\
\hline \multirow[t]{2}{*}{$T T$} & $34(6.4)$ & $44(8.4)$ & $0.75(0.45-1.24)$ & $0.74(0.41-1.32)$ \\
\hline & & & $P$-trend & 0.83 \\
\hline$G T+T T$ & $252(48.2)$ & $248(47.6)$ & $0.95(0.78-1.16)$ & $0.98(0.78-1.22)$ \\
\hline
\end{tabular}

\footnotetext{
* HWE p-value for total NHS and HPFS controls is 0.50 (NHS cancer: HWE $\mathrm{p}=0.86$ controls, $\mathrm{p}=0.05$ cases; HPFS cancer: HWE $\mathrm{p}=0.36$ controls, $\mathrm{p}=0.04$ cases; NHS adenoma: HWE $\mathrm{p}=0.50$ controls, HWE $\mathrm{p}=0.26$ )

1 Conditional logistic regression conditioned on the strata defined by the matching variables

2 Conditional logistic regression conditioned on the strata defined by the matching variables, and adjusted for family history of colon cancer, pack years smoked, body mass index, PMH use, BMI, aspirin intake, physical activity, alcohol intake, total folate consumption, and red meat consumption.

${ }^{3} \mathrm{P}$ for heterogeneity 0.52 for the association in women and men

${ }^{4}$ Armitage test for trend
} 
Hazra et al.

Page 9

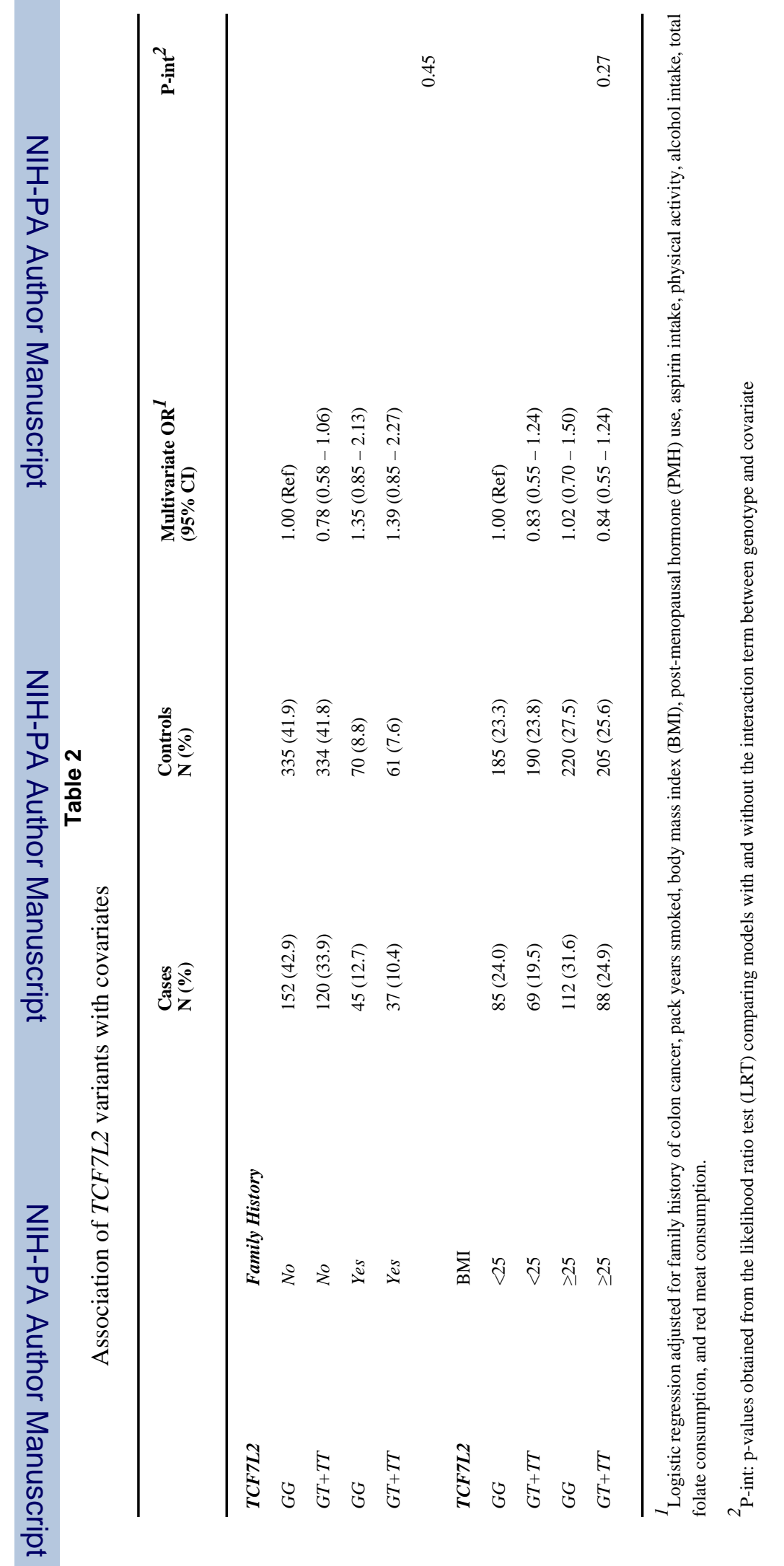

Cancer Causes Control. Author manuscript; available in PMC 2009 November 1. 
Hazra et al.

Page 10

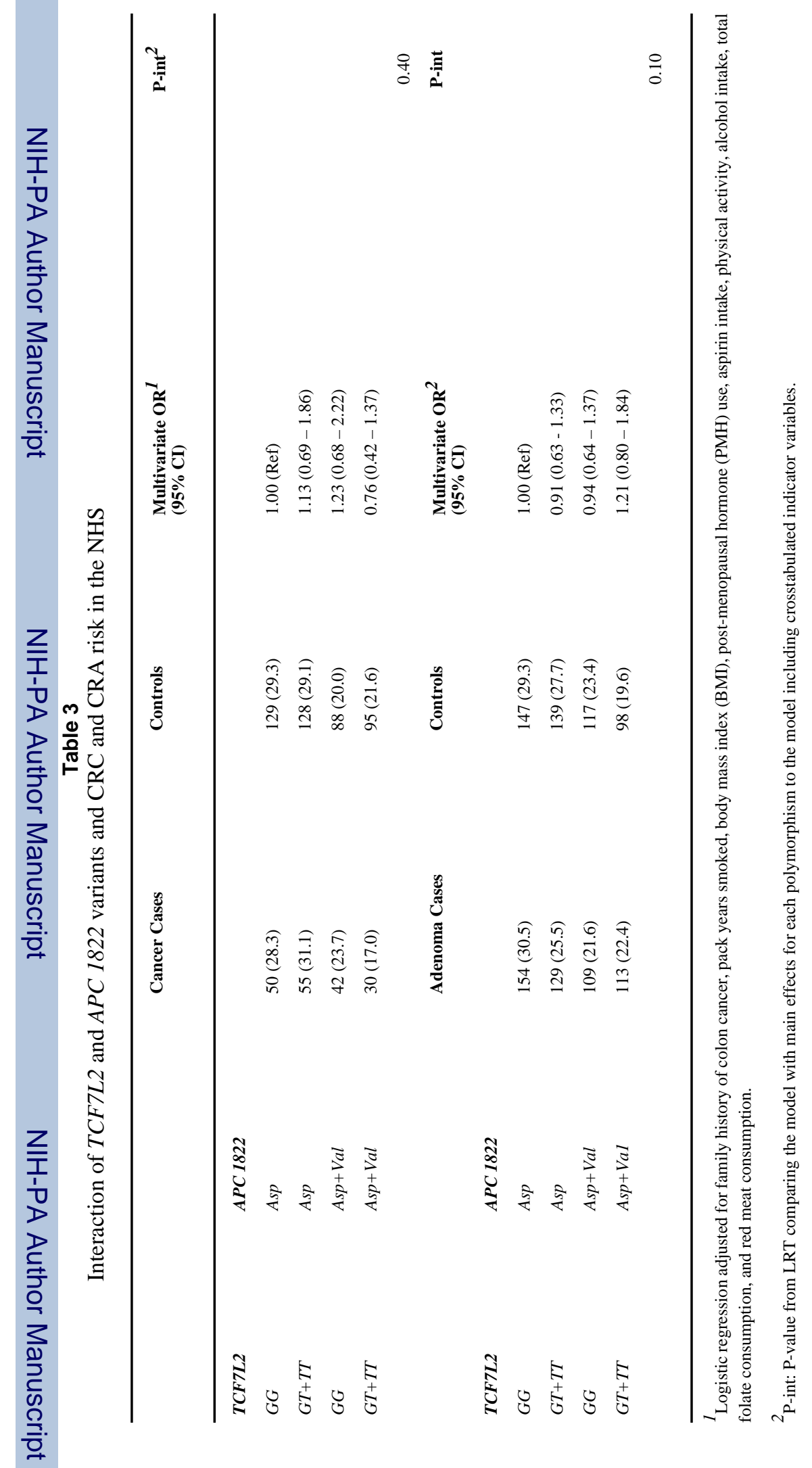

Cancer Causes Control. Author manuscript; available in PMC 2009 November 1. 